\title{
Análisis preliminar de la fitotoxicidad del material particulado sedimentable de la zona urbana de Cuenca
}

\author{
Nancy García Alvear', Mónica Abad Terán ${ }^{2}$ \\ ${ }^{1}$ Centro de Estudios Ambientales, Universidad de Cuenca, Campus Balzay, Av. Víctor Manuel \\ Albornoz, Cuenca, Ecuador. \\ ${ }^{2}$ Facultad de Medicina, Universidad Católica de Cuenca, Pío Bravo y Manuel Vega, Cuenca, \\ Ecuador.
}

Autor para correspondencia: nancy.garcia@ucuenca.edu.ec

Fecha de recepción: 11 de noviembre de 2014 - Fecha de aceptación: 2 de marzo de 2015

\section{RESUMEN}

Este artículo informa sobre la valoración de la fitotóxicidad del Material Particulado Sedimentable (MPS) en la zona urbana del cantón Cuenca. El MPS se recolectó en tres sitios de la zona urbana, mediante el método descrito en el Texto Unificado de Legislación Ambiental Secundaria del Ministerio del Ambiente (TULASMA). Por el material particulado se realizó la determinación de la concentración de los componentes utilizando el análisis gravimétrico, la de los metales pesados por espectroscopía de absorción atómica, y la de aniones por método adaptado de referencia EPA 300.1. Posteriormente se evaluó su fitotóxicidad en cuatro especies, mediante el bioensayo de germinación y elongación de raíz e hipocótilo, en semillas de Lactuca sativa, Brassica oleracea itálica, Allium cepa y Raphanus sativus. En el bioensayo las semillas se expusieron a diferentes diluciones de la suspensión acuosa de MPS. Los resultados muestran que el efecto de los MPS dependió de la concentración y de la especie analizada, evidenciando diferentes grados de fitotóxicidad.

Palabras clave: Fitotóxicidad, material particulado sedimentable, bioensayo.

\begin{abstract}
This paper reports on the phytotoxicity of Sedimentable Particulate Material (acronym in Spanish: MPS) in the urban area of Cuenca. MPS was collected at three sites in the city of Cuenca applying the method described in the Unified Text of Secondary Environmental Legislation of the Ministry of Environment (acronym in Spanish: TULASMA). The concentration of components in the sedimentable particulates was measured using gravimetric analysis, atomic absorption spectroscopy for heavy metals, and a method adopted from the EPA 300.1 reference for anions. The phytotoxicity of MPS was evaluated in bioassays measuring respectively the germination, and the root and hypocotyl elongation of seeds of the following plants: Lactuca sativa, Brassica oleracea italica, Allium cepa, and Raphanus sativus. In the bioassay seeds were exposed to different dilutions of the aqueous suspension of MPS. Results clearly revealed that the effect is concentration and species dependent, showing different levels of phytotoxicity.
\end{abstract}

Keywords: Phytotoxicity, sedimentable particulate material, bioassay.

\section{INTRODUCCION}

El Material Particulado Sedimentable (MPS) está constituido por partículas de diferentes tamaños y composición química, dispersas en la atmósfera. Puede estar suspendido desde horas hasta días, y transportarse largas distancias. Afecta al desarrollo normal de la vida y su peligrosidad depende de su 
composición, tamaño y permanencia en el aire. Su naturaleza, en función de sus características físicas y químicas es compleja; estas características se las considera como factores críticos de toxicidad que intervienen en los diferentes niveles tróficos. Según la Organización Mundial de la Salud (OMS), la contaminación atmosférica causa alrededor de dos millones de muertes prematuras al año en todo el mundo, se estima que el nivel de gravedad de los desórdenes en la salud está directamente relacionado con la concentración de partículas en el aire ambiente (Venegas \& Martín, 2004). La fitotóxicidad es un efecto nocivo o dañino de una sustancia química que se puede expresar en distintos órganos en la planta. Dicho efecto, se manifiesta a través de síntomas como inhibición de la germinación de semillas, reducción del crecimiento de la raíz y la planta en general, enrollamiento foliar, manchas, clorosis y necrosis internerval, lesiones, caída de flores y frutos y reducción de la producción.

Algunos metales como $\mathrm{Zn}, \mathrm{Ni}, \mathrm{Cu}, \mathrm{Mn}, \mathrm{Se}$ y Fe son micronutrientes esenciales para las plantas, porque se requieren en pequeñas cantidades de 1 a 200 ppm, dependiendo del metal y la especie de planta, para su desarrollo normal. Sin embargo, cuando se encuentran en los ecosistemas en altas concentraciones pueden afectar la viabilidad y las funciones celulares (Sierra et al., 2010). La toxicidad que ejercen los metales pesados sobre los cultivos y su biodisponibilidad, puede resultar peligrosa, porque tienden a bioacumularse. Los principales blancos de toxicidad de los metales son proteínas, muchas de ellas con actividad enzimática, afectando a diversos procesos bioquímicos y la integridad de las membranas celulares y sus organelos. Los efectos tóxicos de los metales se ejercen, salvo pocas excepciones, por interacciones entre el ion metálico libre y su blanco. La relación de toxicidad para algunos metales en condiciones in vitro se ha correlacionado de manera directa con la fuerza para unirse en grupos funcionales correspondientes a los Tioles (-SH) (Vargas et al., 2007).

Por otro lado, algunos aniones como nitratos, sulfatos y fosfatos son macronutrientes esenciales porque se necesitan en cantidades relativamente grandes ( 1 a $6 \%$ del peso seco) para el normal desarrollo de las plantas. Sin estas sustancias, el crecimiento de la planta se retarda. Sin embargo, demasiado fósforo o una abundancia de nitratos tienen un efecto negativo en plantas y animales (Sierra et al., 2010).

Un bioensayo o prueba de toxicidad, es una técnica empleada para determinar si un residuo químico presente en el ambiente está en cantidades suficientemente altas para afectar adversamente algunos aspectos del espectro normal de actividades de animales y plantas. Se realizan con el supuesto de que los organismos probados son "sucedáneos" o "claves" de organismos "superiores" presentes en su ambiente natural. La ecotoxicología está basada en el principio de que hay una relación directa entre la reacción tóxica (la respuesta) y la cantidad de sustancia recibida (la dosis). Un supuesto importante de esta relación es que existe una dosis a la cual no ocurre respuesta, el segundo supuesto es que una vez que la dosis máxima ha sido alcanzada cualquier incremento en ésta no resultará en un incremento del efecto (Escalante, 2000).

Las plantas vasculares, como por ejemplo hortalizas, cereales y leguminosas, han sido recomendadas por la Agencia de Protección al Medioambiente (EPA) y por la Administración de Drogas y Alimentos (FDA), debido a su buena sensibilidad, en comparación con semillas de otras especies de plantas terrestres. El ensayo ecotoxicológico con semillas de plantas vasculares presenta algunas ventajas sobre otras especies terrestres, lo que permite usar cuantitativamente el crecimiento de la raíz (Wang, 1987). Los efectos fitotóxicos se determinan por la inhibición en la germinación de las semillas, y la inhibición en la elongación de la radícula e hipocótilo, puesto que en el periodo de germinación y los primeros días de desarrollo de la plántula ocurren numerosos procesos fisiológicos en los que la presencia de una sustancia tóxica puede interferir alterando la supervivencia y el desarrollo normal de la planta, siendo por lo tanto una etapa de gran sensibilidad frente a factores externos adversos. La inhibición en la elongación de la radícula e hipocótilo constituyen indicadores subletales muy sensibles para la evaluación de efectos biológicos en vegetales, aportando información complementaria a la proporcionada al estudiar el efecto en la germinación (Sobrero \& Ronco, 2005).

Esta investigación se fundamentó en la información obtenida en investigaciones anteriores (García \& Cooman, 2004; García et al., 2006; Jachero \& Moscoso, 2006) que establecen que el aire de la zona urbana de Cuenca presenta altos niveles de contaminantes entre los que se encuentra el material particulado, y un alto contenido de metales pesados. Planteándose como hipótesis que el 
material particulado sedimentable (MPS) generado en la zona urbana de Cuenca, contiene sustancias que causan un efecto fitotóxico importante.

\section{MATERIALES Y MÉTODOS}

\subsection{Muestreo de MPS}

Se realizó el muestreo en tres sectores de la zona urbana de Cuenca. La selección de los sitios de muestreo se hizo en base a los estudios previos (García \& Cooman, 2004; García et al., 2006; Moscoso \& Pacheco, 2006) que los determinan como puntos de altos niveles de contaminación, y presumiendo en función de dichas investigaciones, que estos tres puntos constituyen una muestra representativa de la ciudad. Estos sitios fueron: Av. Loja y Remigio Romero, Hermano Miguel y Vega Muñoz, y Av. Octavio Chacón (parque industrial). El tiempo de muestreo fue de 30 días continuos, desde el 16 de febrero al 17 de marzo del 2013, en cada punto, en forma simultánea; colocándose 4 envases abiertos con una medida de $9.2 \mathrm{~cm}$ de radio interno superior, con un área de captación del envase de $265.9 \mathrm{~cm}^{2}$, y una altura de $24.7 \mathrm{~cm}$. Los envases para la recolección del MPS, se ubicaron a una altura de 3 metros sobre el nivel del suelo. Terminado el período de monitoreo, los envases se transportaron al laboratorio, protegidos de la luz y cubiertos para evitar la pérdida de muestra.

Se realizó el cálculo de concentración de MPS por análisis gravimétrico, de acuerdo al método de medición de concentraciones de contaminantes comunes del aire, descritos en el Libro VI, Anexo 4: Norma de Calidad del Aire Ambiente (TULASMA, 2002). Las partículas recolectadas se clasificaron en solubles e insolubles. Las partículas insolubles se determinaron por diferencia de peso ganado por un filtro de $47 \mathrm{~mm}$ (filtros de microfibras de cuarzo); las partículas solubles se determinaron mediante diferencia de peso ganado en una cápsula de porcelana, en la cual se evaporó el líquido de lavado del envase. La concentración total de partículas sedimentables es la suma de partículas solubles e insolubles, normalizadas con respecto al área total de captación del envase.

\subsection{Población para el bioensayo}

La población del estudio consistió en una serie de semillas de las siguientes especies: Lactuca sativa (lechuga), Brassica oleracea itálica (brócoli), Allium cepa (cebolla) y Raphanus sativus (rábano). Se adquirió 2 onzas de semillas de cada una de las especies en estudio, en la distribuidora COMSERVAGRO de semillas certificadas BONANZA SEEDS, producto de las EE.UU., con las siguientes especificaciones: pureza: $99.9 \%$; germinación: superior a $85 \%$; materia inerte: inferior a 10\%; fecha de validez: del 12/2010 hasta 12/2014. Se tomaron 20 semillas por cada dilución, y se trabajó por duplicado (40 semillas por cada dilución).

\subsection{Caracterización del MPS}

El método analítico empleado para determinar la presencia de metales fue el NIOSH Método 7082 (Atomic Absorption Spectrometry), que en resumen contempla el ataque ácido y completa solubilización de los metales, aforo a un volumen conocido, homogeneización y lectura en el equipo de absorción atómica (previamente calibrado empleando los patrones certificados por el NIST y dentro del rango lineal), utilizando las respectivas lámparas. Para la determinación de aniones se empleó el método adaptado de referencia EPA 330.1, que emplea un volumen determinado de muestra acuosa, usualmente $5 \mathrm{ml}$ son filtrados por un microfiltro de celulosa de $0.45 \mu \mathrm{m}$. Un mililitro de este filtrado es analizado con cromatografía líquida de alta eficiencia con una columna en fase de reversa. Para cualificar y cuantificar se elaboraron curvas de calibración de diferentes rangos. 


\subsection{Preparación de las suspensiones acuosas del MPS}

De los tres puntos de monitoreo y recolección, se procedió a la preparación de una suspensión acuosa al $4 \%$ con agua desionizada y de ésta se preparó 5 diluciones, respectivamente $2 \% ; 0.2 \% ; 0.02 \%$; $0.002 \%$; y $0.0002 \%)$, y un control negativo $(0 \%)$.

\subsection{Bioensayo de fitotóxicidad aguda con semillas}

Se trata de una prueba estática de toxicidad aguda en 120 horas de exposición, conforme con el método adaptado por Sobrero \& Ronco (2005) de la Guía 850.4200 US EPA-OPPTS. Previo a la implementación de la prueba, se verificó que cada lote de semillas tenga un porcentaje de germinación superior al 90\%. Se evaluó los efectos fitotóxicos del MPS en el proceso de germinación de las semillas y en el desarrollo de las plántulas - radícula e hipocótilo - durante los primeros cinco días de crecimiento. Para la evaluación de los efectos fitotóxicos, se determinó la inhibición en la germinación y la inhibición o estimulación en la elongación de la radícula e hipocótilo.

\subsection{Siembra de las semillas}

Se realizó en cajas de Petri, utilizando papel de filtro Whatman, grado 3, como soporte y medio de imbibición. El volumen de muestra para el ensayo fue de $5 \mathrm{ml}$ por caja, empleando agua destilada en los controles negativos y como medio de dilución. Por cada caja de Petri, y una vez embebido el papel de filtro, se colocaron 20 semillas, teniendo en cuenta el dejar espacio suficiente entre las semillas para permitir la elongación de las raíces. Las cajas se taparon para evitar la pérdida de humedad, y se cubrieron de la luz inmediatamente después de iniciada la imbibición y durante el periodo de ensayo para facilitar la germinación. Se incubó por un periodo de 120 horas a $22^{\circ} \mathrm{C}$. Se realizó una réplica para cada dilución ensayada. Transcurridas las 120 horas se hizo las mediciones y observaciones de las plántulas, registrando todos los datos, para el análisis estadístico. La Figura 1 evidencia mediante una fotografía el desarrollo de las semillas de rábano luego de 120 horas.

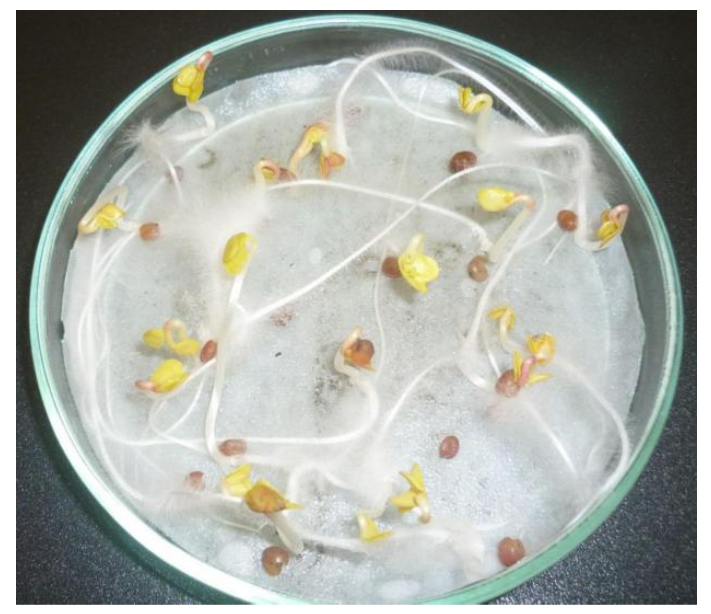

Figura 1. Fotografía de cajas de Petri con semillas de rábano, luego de 120 horas de la siembre (final de la prueba).

\subsection{Mediciones efectuadas}

Se registró el número de semillas que germinaron normalmente, respecto del total de semillas colocadas al inicio del ensayo, considerando como criterio de germinación la aparición visible de la radícula. Utilizando una regla se midió cuidadosamente la longitud de la radícula e hipocótilo de cada una de las plántulas correspondientes a los controles y a cada concentración de MPS. La medida de elongación de la radícula se considera desde el nudo (región más engrosada de transición entre la radícula e hipocótilo) hasta el ápice radicular. La medida de elongación del hipocótilo se considera desde el nudo hasta la inserción de los cotiledones. 


\subsection{Análisis estadístico}

Todas las pruebas se realizaron por duplicado y utilizando un control. El análisis estadístico se lo hizo mediante el análisis de varianza (ANOVA). Se utilizó el programa estadístico SPSS versión 19.

\section{RESULTADOS Y DISCUSIÓN}

\subsection{Concentración de MPS}

La Tabla 1 muestra las concentraciones de MPS obtenidas en los tres puntos de monitoreo. Se observa que en la zona 1 la concentración es de $1.11 \mathrm{mg} \mathrm{cm}^{-2}$ x $30 \mathrm{~d}$ y en la zona 3 es de $1.2 \mathrm{mg} \mathrm{cm}^{-2}$ x $30 \mathrm{~d}$, concentraciones superiores a lo que determina la norma ecuatoriana mediante su legislación (TULASMA, 2002), cuyo valor límite es de $1 \mathrm{mg} \mathrm{cm}^{-2}$ x $30 \mathrm{~d}$. En la zona 2 la concentración obtenida es de $0.56 \mathrm{mg} \mathrm{cm}^{-2}$ x $30 \mathrm{~d}$, valor que está por debajo del valor máximo especificado en ésta normativa. Sin embargo, haciendo referencia a la normativa de la OMS que es mas exigente, y establece $0.5 \mathrm{mg}$ $\mathrm{cm}^{-2}$ x $30 \mathrm{~d}$, la concentración en los tres puntos de monitoreo supera el valor máximo permitido. Los resultados indicarían que las mayores concentraciones de MPS se presentan en zonas que no están en el centro histórico de la ciudad, corroborando los resultados de otros estudios (García et al., 2006).

Tabla 1. Concentraciones de MPS $\left(\mathrm{mg} \mathrm{cm}^{-2}\right.$ x $\left.30 \mathrm{~d}\right)$ en los tres puntos de monitoreo en la ciudad de Cuenca.

\begin{tabular}{cccc}
\hline Dirección & Latitud & Longitud & MPS $\left(\mathrm{mg} \mathrm{cm}^{-2} \times 30 \mathrm{~d}\right)$ \\
\hline Avenida Loja & $2^{\circ} 54^{\prime} 02.91^{\prime \prime} \mathrm{S}$ & $79^{\circ} 0^{\prime} 52.30^{\prime \prime} \mathrm{O}$ & 1.11 \\
Hermano Miguel & $2^{\circ} 53^{\prime} 39.31^{\prime \prime} \mathrm{S}$ & $79^{\circ} 0^{\prime} 4.62^{\prime \prime} \mathrm{O}$ & 0.56 \\
Parque Industrial & $2^{\circ} 55^{\prime} 38, " \mathrm{~S}$ & $78^{\circ} 58^{\prime} 35.75^{\prime \prime} \mathrm{O}$ & 1.20 \\
\hline
\end{tabular}

Tabla 2. Composición del material particulado (MPS) en lo referente a metales pesados y aniones. Valores promedio de los tres puntos de monitoreo.

\begin{tabular}{cccccccccc}
\hline & \multicolumn{4}{c}{ Metales pesados $(\mathrm{ppm})$} & \multicolumn{4}{c}{ Aniones $\left(\mathrm{mg} \mathrm{kg}^{-1}\right)$} \\
$\mathrm{Cu}$ & $\mathrm{Cd}$ & $\mathrm{Cr}$ & $\mathrm{Fe}$ & $\mathrm{Mn}$ & $\mathrm{Ni}$ & $\mathrm{Zn}$ & Cloruro & Nitrato & Sulfato \\
\hline 4.36 & $\mathrm{NC}$ & 149.63 & 26,081 & 541.71 & 128 & 952.38 & 665 & $>4$ & 6,311 \\
\hline
\end{tabular}

\subsection{Caracterización química del MPS}

La Tabla 2 expone la composición de metales pesados y aniones en el MPS recolectado. Estos valores representan la media de los tres puntos de recolección, considerando que sería representativa de la zona urbana (García \& Cooman, 2004; García et al., 2006; Moscoso \& Pacheco, 2006), pues los análisis no se realizaron en cada muestra por separado. En el caso de los metales pesados se observa que los valores más elevados se encuentran en hierro, manganeso, zinc y cromo; el cadmio no se pudo detectar con la técnica empleada. No se puede establecer una comparación con valores referenciales, debido a que la normativa ecuatoriana no establece valores referenciales para aire ambiente, así tampoco las normativas internacionales que contemplan solo valores para el material particulado de tamaño menor como el PM10 (partes por millón con un diámetro menor de $10 \mu \mathrm{m} ; 1$ micrómetro corresponde la milésima parte de 1 milímetro), esto debido a que estas normativas consideran la mayor peligrosidad del material particulado que afecta directamente a la salud humana. Jachero \& Moscoso (2009) en su estudio realizado sobre material particulado en Cuenca, concluyen que el MPS tiene entre 1 y $2 \%$ en peso de metales pesados; mientras que, en la presente investigación, se determinó un 2.8\%. Cabe aclarar que en el estudio antes mencionado, se monitorearon 9 puntos de la ciudad, pudiendo ser esta la razón de la diferencia que se observa con el resultado de la presente investigación. Por otro lado, los valores encontrados actualmente podrían ser un indicador de que la contaminación ha variado en su composición en los últimos años. La evidente presencia de metales pesado en el MPS, estaría indicando su posible fitotóxicidad. En el caso del níquel, este es esencial 
para plantas, animales y bacterias, y es requerido en concentraciones muy bajas $\left(1.7 \mathrm{nmol} \mathrm{g} \mathrm{g}^{-1}\right.$ en biomasa seco); el exceso de níquel es tóxico. Los síntomas de toxicidad se observan entre 0.19 y 0.85 $\mu \mathrm{mol} \mathrm{g}^{-1}$ en peso seco de planta, e incluyen la inhibición del crecimiento de la raíz, y la aparición de clorosis entre las venas de las hojas. Por otro lado, un exceso de zinc, no inhibe la germinación, pero si el crecimiento de la raíz en concentraciones de $7 \mathrm{mM}$ en ciertos tipos de plantas (Vargas et al., 2007).

En el caso de los aniones tampoco es posible comparar con la normativa, pues no existen valores referenciales ni en la normativa ecuatoriana ni en la normativa de la OMS. En el estudio realizado por Ribera \& Canto (2012) en el Centro de Estudios Científicos, para la Compañía Minera Nevada, en los análisis de MPS se determina la concentración de aniones, para cloruros con un rango entre 0.47 a 5.8 $\mathrm{mg} \mathrm{l}^{-1}$; para sulfatos un rango entre 0.37 a $169.79 \mathrm{mg} \mathrm{l}^{-1}$; y para nitratos un rango entre 0.34 a 10.6 $\mathrm{mg} \mathrm{l}^{-1}$. En este caso el análisis fue de los aniones solubles, mientras en el presente estudio se realizó en toda la muestra, por lo que los valores son mucho más altos para cloruros y sulfatos.

Díaz y Paez (2006), en un estudio realizado en Quito, en que se hace una caracterización química en PM10, encontraron los siguientes valores: sulfatos el 13\% del total del material particulado, los nitratos el $6 \%$ y cloruros el $0.25 \%$, señalando que el alto contenido de azufre de los combustibles, provoca altas emisiones de este elemento y material particulado. Los resultados obtenidos en el presente estudio son en MPS y son valores muchos menores.

\subsection{Porcentaje de germinación}

La Tabla 3 presenta el porcentaje de germinación de las semillas de las cuatro especies, en las diferentes concentraciones. La prueba se realizó por duplicado, por tanto constan los resultados de ambas pruebas. El cálculo de los porcentajes de germinación se realizó con la finalidad de comprobar si el MPS puede alterar los procesos de la germinación de semillas.

Tabla 3. Porcentajes de germinación (por duplicado) de las semillas de las cuatro especies, en las diferentes concentraciones de MPS.

\begin{tabular}{lcccccccc}
\hline \multirow{2}{*}{$\begin{array}{c}\text { MPS } \\
(\%)\end{array}$} & \multicolumn{2}{c}{ Lechuga } & \multicolumn{2}{c}{ Porcentaje de germinación } \\
Brócoli & \multicolumn{2}{c}{ Cebolla } & \multicolumn{2}{c}{ Rábano } \\
\cline { 2 - 8 } & Prueba 1 & Prueba 2 & Prueba 1 & Prueba 2 & Prueba 1 & Prueba 2 & Prueba 1 & Prueba 2 \\
\hline 0 & 100 & 95 & 95 & 100 & 90 & 90 & 100 & 95 \\
0.0002 & 95 & 95 & 95 & 95 & 45 & 45 & 100 & 100 \\
0.002 & 100 & 90 & 90 & 95 & 50 & 40 & 100 & 100 \\
0.02 & 100 & 90 & 90 & 100 & 40 & 45 & 100 & 100 \\
0.2 & 100 & 95 & 90 & 95 & 40 & 40 & 100 & 100 \\
2 & 100 & 100 & 95 & 95 & 45 & 55 & 100 & 100 \\
4 & 100 & 100 & 95 & 95 & 50 & 40 & 95 & 100 \\
\hline
\end{tabular}

Se observa que en las tres especies: lechuga, brócoli y rábano, no hay interferencia en la germinación, pues, los porcentajes están de acuerdo a las especificaciones del productor de las semillas (superior al 85\%) y son constantes en todas las pruebas con las distintas concentraciones de MPS. Este resultado coincide con lo que establecen los estudios sobre la prueba tradicional de germinación de semillas. En la que compuestos tóxicos presentes en niveles de concentración bajos no son suficientes para inhibir la germinación, pero que sin embargo pueden retardar o inhibir completamente los procesos de elongación de la radícula o del hipocótilo (Sobrero \& Ronco, 2005). No ocurre lo mismo en el caso de la cebolla, pues se observa una importante inhibición en el porcentaje de germinación, en un promedio del 55\%. Esto haría pensar que la cebolla es una especie más vulnerable a la presencia de este tipo de elementos tóxicos. Una concentración de $0.0002 \%$ de MPS es suficiente para que el porcentaje de germinación disminuya al 45\%. Resultados que coinciden con los obtenidos por Iannconne \& Alvariño (2005), quienes afirman que las especies tienen diferentes sensibilidades que dependen de muchos factores como tiempos de exposición, edad de las semillas y otros. 


\subsection{Elongación de raíz e hipocótilo}

La Tabla 4 presenta el detalle del tratamiento estadístico de los datos con respecto a la longitud de raíz y de hipocótilo.

Tabla 4. Longitud de la raíz (parte superior) e hipocótilo (parte inferior) en $\mathrm{cm}$ por especie de planta en función de la concentración del MPS.

\begin{tabular}{lcccccccc}
\hline & \multicolumn{1}{c}{ Lechuga } & \multicolumn{3}{c}{ Longitud de la raíz en cm } \\
MPS $(\%)$ & \multicolumn{1}{c}{ Brócoli } & \multicolumn{2}{c}{ Cebolla } & \multicolumn{2}{c}{ Rábano } \\
\cline { 2 - 9 } & Media & DT & Media & DT & Media & DT & Media & DT \\
\hline 0 & $1.30 \mathrm{a}$ & 0.54 & $2.70 \mathrm{~b}$ & 1.11 & $0.20 \mathrm{~b}$ & 1.123 & $7.45 \mathrm{~b}$ & 3.34 \\
0.0002 & $1.27 \mathrm{a}$ & 0.48 & $3.54 \mathrm{~b}$ & 1.68 & $0.10 \mathrm{a}$ & 0.115 & $8.44 \mathrm{~b}$ & 2.68 \\
0.002 & $1.36 \mathrm{a}$ & 0.56 & $3.11 \mathrm{~b}$ & 1.57 & $0.10 \mathrm{a}$ & 0.120 & $8.56 \mathrm{~b}$ & 2.06 \\
0.02 & $1.31 \mathrm{a}$ & 0.67 & $3.23 \mathrm{~b}$ & 1.59 & $0.10 \mathrm{a}$ & 0.133 & $7.92 \mathrm{~b}$ & 2.59 \\
0.2 & $1.39 \mathrm{a}$ & 0.52 & $2.71 \mathrm{~b}$ & 1.53 & $0.09 \mathrm{a}$ & 0.125 & $8.32 \mathrm{~b}$ & 2.26 \\
2 & $2.31 \mathrm{~b}$ & 0.67 & $2.78 \mathrm{~b}$ & 1.48 & $0.11 \mathrm{ab}$ & 0.135 & $7.10 \mathrm{~b}$ & 2.92 \\
4 & $0.69 \mathrm{c}$ & 0.34 & $1.16 \mathrm{a}$ & 0.76 & $0.12 \mathrm{ab}$ & 0.147 & $4.19 \mathrm{a}$ & 1.64 \\
\hline
\end{tabular}

\begin{tabular}{lcccccccc}
\hline & \multicolumn{1}{c}{ Lechuga } & \multicolumn{2}{c}{$\begin{array}{c}\text { Longitud de la hipocótilo en cm } \\
\text { Crócoli }\end{array}$} & \multicolumn{2}{c}{ Cebolla } & \multicolumn{2}{c}{ Rábano } \\
\cline { 2 - 9 } & Media & DT & Media & DT & Media & DT & Media & DT \\
\hline 0 & $1.67 \mathrm{ab}$ & 0.73 & $1.97 \mathrm{~b}$ & 0.90 & $0.18 \mathrm{~b}$ & 0.22 & $2.23 \mathrm{a}$ & 0.992 \\
0.0002 & $1.76 \mathrm{~b}$ & 0.74 & $2.03 \mathrm{~b}$ & 1.04 & $0.09 \mathrm{ab}$ & 0.18 & $2.15 \mathrm{a}$ & 0.75 \\
0.002 & $1.71 \mathrm{~b}$ & 0.73 & $1.95 \mathrm{~b}$ & 1.06 & $0.08 \mathrm{ab}$ & 0.16 & $1.94 \mathrm{a}$ & 0.63 \\
0.02 & $1.68 \mathrm{ab}$ & 0.84 & $1.79 \mathrm{~b}$ & 0.87 & $0.09 \mathrm{ab}$ & 0.18 & $1.90 \mathrm{a}$ & 0.78 \\
0.2 & $1.73 \mathrm{~b}$ & 0.60 & $1.67 \mathrm{~b}$ & 1.00 & $0.14 \mathrm{~b}$ & 0.21 & $2.14 \mathrm{a}$ & 0.69 \\
2 & $2.15 \mathrm{~b}$ & 0.60 & $2.00 \mathrm{~b}$ & 0.95 & $0.10 \mathrm{ab}$ & 0.20 & $2.28 \mathrm{a}$ & 0.86 \\
4 & $1.19 \mathrm{a}$ & 0.53 & $0.95 \mathrm{a}$ & 0.67 & $0.00 \mathrm{a}$ & 0.00 & $1.73 \mathrm{a}$ & 0.65 \\
\hline
\end{tabular}

Leyenda:

- Media de la prueba 1 y 2

- DT = Desviación típica de la prueba 1 y 2

- Promedios en una misma columna, seguidos por la misma letra, no difieren significativamente según la Prueba de Tukey $(\mathrm{p}<0.05)$

La Figura 2 ilustra la elongación de la raíz en las semillas de las cuatro especies, lechuga, brócoli, cebolla y rábano. Las concentraciones muy bajas en lechuga, rábano y brócoli generan una estimulación, sin embargo cuando se incrementa la concentración, llegando al 4\% se evidencia la inhibición. Concentraciones mayores al $0.2 \%$ provocan en el rábano una drástica inhibición, mayor a la presentada en las otras especies. Para el caso de la cebolla, en las semillas que germinaron, se presentó una inhibición total del crecimiento radicular. En el brócoli, luego de la estimulación en $0.0002 \%$, se da una paulatina disminución del crecimiento hasta el $2 \%$, luego de lo cual, la pendiente cae de forma drástica al 4\%. Llama la atención la estimulación del crecimiento que se produjo con la lechuga, en una concentración del 2\%. Dado que las pruebas se hicieron por duplicado, se descarta la posibilidad de que se trate de un error experimental. Sin embargo, a mayores concentraciones (4\%) se produjo la inhibición del crecimiento.

La explicación parte del hecho de que sulfatos y algunos metales, son nutrientes esenciales para el normal desarrollo de las plantas (Sierra et al., 2010). Sin embargo cuando se encuentran en los ecosistemas en altas concentraciones pueden afectar la viabilidad y las funciones celulares. (Vargas \& col., 2007). Giardina et al. (2012) observan un efecto estimulante del cromo a bajas concentraciones para tallo y radícula, en el caso del fréjol, afirmando que estudios anteriores (Giardina et al., 2010) en lechuga, tomate y rabanito, concentraciones entre 0.01 y $0.001 \mathrm{mM}$, resultaron estimulantes del crecimiento de las tres especies.

En el caso de la cebolla, nuevamente se ve un comportamiento diferente, podría ser que esta especie tiene un requerimiento nutricional particular, o es más sensible a alguno de los elementos presentes en el MPS. Aurazo \& Esparza (1995) encontraron que el cromo (VI) afecta el crecimiento 
radicular de la cebolla: en concentraciones de $1 \mathrm{mg} \mathrm{l}^{-1}$ se inhibe en un $25 \%$, y en concentraciones entre 5 y $70 \mathrm{mg} \mathrm{l}^{-1}$ se inhibe entre un 75 y $85 \%$, y en valores superiores a $70 \mathrm{mg} \mathrm{l}^{-1}$ se inhibe en un $100 \%$.

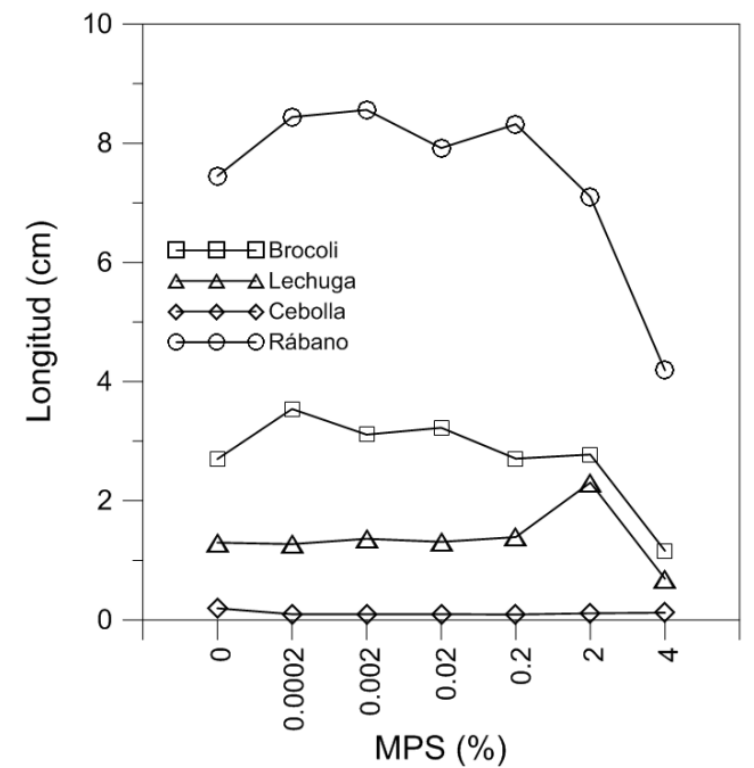

Figura 2. Comparación del promedio de la elongación de la raíz en las cuatro especies vegetales en diferentes concentraciones de MPS.

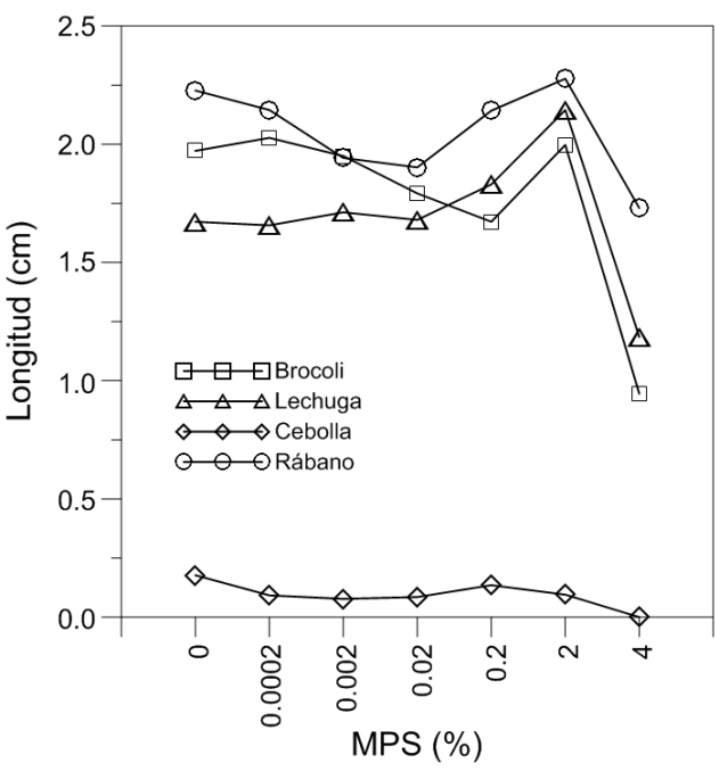

Figura 3. Comparación del promedio de la elongación del hipocótilo en las cuatro especies vegetales en diferentes concentraciones de MPS.

La Figura 3 muestra los resultados de elongación del hipocótilo en las cuatro especies. A medida que se incrementa la concentración de MPS en lechuga, brócoli y rábano se evidencia la inhibición. Hay una curiosa semejanza de las curvas en las tres especies, que presentan una recuperación de crecimiento en la concentración de $2 \%$. En la cebolla, además de inhibirse la germinación y crecimiento radicular, se da una inhibición del crecimiento de hipocótilo. Estos resultados confirman el hecho de que, durante el periodo de germinación y los primeros días de desarrollo de la plántula ocurren numerosos procesos fisiológicos en los que la presencia de una sustancia tóxica puede interferir alterando la supervivencia y el desarrollo normal de la planta, siendo por lo tanto una etapa de gran sensibilidad frente a factores externos adversos. (Sobrero \& Ronco, 2005).

La evaluación del desarrollo de la radícula e hipocótilo constituye un indicador para determinar la capacidad de establecimiento y desarrollo de la planta. A diferencia de la prueba tradicional de germinación de semillas, la evaluación del efecto en la elongación de la radícula e hipocótilo de las plántulas permite ponderar el efecto tóxico de compuestos solubles presentes en niveles de concentración tan bajos que no son suficientes para inhibir la germinación, pero que sin embargo pueden retardar o inhibir completamente los procesos de elongación de la radícula o del hipocótilo.

Los resultados obtenidos muestran una relación con el estudio de Sobrero (2010), quien concluye que existen diferentes respuestas entre distintas especies de plantas vasculares respecto de los niveles de tolerancia o toxicidad frente a la exposición a metales pesados. Además, Wang (1987) menciona, que compara la respuesta de tres especies de plantas vasculares Lactuca sativa (lechuga), Cucumis sativus (pepino) y Panicum miliaceum (sorgo) a compuestos orgánicos y metales pesados, observando una mayor sensibilidad de L. sativa por los metales, mientras que con relación a la toxicidad de compuestos orgánicos, la sensibilidad fue mayor en la especie $P$. miliaceum. Cheung et al. (1989) comparan la sensibilidad de 13 especies comestibles frente a la exposición a diferentes metales pesados, observando que la respuesta de éstas es dependiente del elemento estudiado y no encuentran patrones generales de toxicidad. Arambašić et al. (1995) observan una mayor sensibilidad de Allium cepa (cebolla) respecto de Lepidium sativum (berro) al evaluar el efecto agudo del $\mathrm{Cu}$ (II), $\mathrm{Zn}$ (II) y $\mathrm{Pb}$ (II) en la elongación de la raíz. 
Por otra parte, los resultados de la evaluación de la toxicidad de contaminantes utilizando semillas, también indican una amplia variedad en la respuesta a los metales (Wang, 1987). Prieto et al. (2009) en su estudio de contaminación y fitotóxicidad en plantas por metales pesados provenientes de suelo y agua, señalan la potencialidad del uso de cebolla, betarraga, arroz y rabanito para evaluar la toxicidad y el riesgo de sustancias químicas peligrosas en el ambiente. Además manifiesta que todas las plantas absorben metales del suelo donde se encuentran, pero en distinto grado, dependiendo de la especie vegetal y de las características y contenido en metales del suelo. Sin embargo, el efecto de metales pesados sobre plantas vasculares no se conoce bien. Las diferentes respuestas de las plantas vasculares a metales pesados pueden ser atribuidas a factores genéticos y fisiológicos. Metales como el plomo $(\mathrm{Pb})$ reducen el crecimiento radicular y la frecuencia de células mitóticas y el incremento de la frecuencia de células aberrantes en cebolla. La intensidad del efecto está en función de la concentración del $\mathrm{Pb}$.

Tabla 5. Efecto del MPS en las cuatro especies vegetales, expresado en porcentajes media de estimulación-inhibición del crecimiento de raíz e hipocótilo.

\begin{tabular}{lcccccc}
\hline \multirow{2}{*}{ Especie } & \multirow{2}{*}{ Porcentaje de crecimiento } & \multirow{2}{*}{ MPS $(\%)$} & \multicolumn{2}{c}{$\%$ de inhibición al 4\% de } & \multirow{2}{*}{ Fitotóxicidad } \\
\cline { 2 - 3 } \cline { 5 - 6 } & Raíz & Hipocótilo & & Raíz & Hipocótilo & \\
\hline Lechuga & 77.65 & 28.25 & 2 & 46.82 & 29.15 & moderada \\
Brócoli & 31.11 & 2.78 & 0.0002 & 57.04 & 52.05 & severa \\
Cebolla & 0 & 0 & todas & 38.47 & 100 & severa \\
Rábano & 13.25 & 14.86 & 0.002 & 47.78 & 22.34 & moderada \\
\hline
\end{tabular}

\subsection{Evaluación de la fitotóxicidad}

Utilizando el método de Zucconi (Varnero et al., 2007) que es el más utilizado para determinar los niveles de fitotóxicidad. Clasificando la fitotóxicidad de acuerdo al porcentaje medio de inhibición de raíz e hipocótilo en moderada, media y severa, se obtuvo los resultados de la Tabla 5, que muestra los porcentajes de estimulación del crecimiento, a diferentes concentraciones de MPS para cada especie, así en la lechuga, se observa una estimulación hasta el 2\%, mientras que en el brócoli se da solamente en la concentración más baja del $0.0002 \%$ y en el rábano en el $0.002 \%$. No presentándose efecto estimulante alguno en el caso de la cebolla. Los porcentajes de inhibición con un $4 \%$ de MPS, muestran una inhibición severa para brócoli y cebolla, y moderada para lechuga y rábano.

\section{CONCLUSIONES}

Los niveles de MPS en la ciudad de Cuenca superan la normativa de la OMS, y su composición en metales pesados es importante, siendo hierro, cromo, manganeso y zinc los más significativos. En los resultados del bioensayo se demostró que el MPS, altera el crecimiento normal de la raíz e hipocótilo en las cuatro especies analizadas. Además de inhibir en un 55\% la germinación en la cebolla, por lo que se evidencia el efecto tóxico del MPS sobre el normal desarrollo en las primeras etapas de crecimiento de una planta. Concentraciones bajas de MPS tienen un efecto estimulante en el crecimiento de lechuga, rábano y brócoli, debido al aporte de micronutrientes para su desarrollo. Efecto que se manifiesta a diferentes concentraciones para cada especie.

La cebolla es una especie altamente sensible a compuestos tóxicos, como lo demuestra el bajo porcentaje de germinación, así como la considerable inhibición del crecimiento radicular y del hipocótilo, aun a bajas concentraciones de MPS. No se conoce con certeza, cual es el componente del MPS específico que le afecta más que a las otras especies, y sería objeto de nuevos estudios, realizando bioensayos con sustancias puras que sean componentes del MPS, para comparar los resultados de cada una de ellas y encontrar la que produce el mayor efecto inhibitorio.

El MPS evaluado a una concentración de 4\%, tiene un efecto fitotóxico severo para las especies de brócoli y cebolla, y moderado para las especies de lechuga y rábano. 


\section{AGRADECIMIENTOS}

Las autoras agradecen el apoyo del Centro de Estudios Ambientales (CEA) de la Universidad de Cuenca por facilitar su laboratorio e infraestructura para este trabajo.

\section{BIBLIOGRAFIA}

Arambašić, S., S. Bejelic, G. Subakov, 1995. Acute toxicity of heavy metals (copper, lead, zinc), phenol and sodium on Allium cepa L., Lepidium sativum L. and Daphnia magna St.: Comparative investigations and the practical applications. Water Res., 29(2), 497-503.

Aurazo, M., M.L. Esparza, 1995. Toxicidad aguda del cromo usando Allium cepa. OPS/CEPIS/95-25 LAB. Descargado de www.bvsde.paho.org/bvsacd/scan2/039219/039219.pdf en octubre de 2014.

Cheung, I.H., M.H. Wong, N.F.I. Tam, 1989. Root and shoot elongation as an assessment of heavy metal toxicity and $\mathrm{Zn}$ equivalent value of edible crops. Environmental Bioassay Techniques and their Application Developments in Hydrobiology, 54, 377-383.

Díaz, V., C., Páez, 2006. Contaminación por material particulado en Quito y caracterización química de las muestras. Corporación para el mejoramiento del aire en Quito. Quito, Ecuador. Acta Nova, 3(2), 308-322. Descargado de http://www.ucbcba.edu.bo/Publicaciones/revistas/actanova/ documentos/v3n2/v3.n2.diaz.pdf en octubre de 2014.

Escalante, E., 2000. Estudio de ecotoxicidad de un suelo contaminado con hidrocarburos. Universidad Autónoma Metropolitana de Iztapalapa. Descarga de http://www.148.206.53.84/tesiuami/ UAMI10492.pdf en septiembre de 2014.

García, N., G. Carrillo, A. Astudillo, V. Vázquez, 2006. Niveles de material particulado en diferentes zonas de la ciudad de Cuenca. En: Calidad de aire. Centro de Estudios Ambientales de la Universidad de Cuenca (http://www.ceaucuenca.ec/index.php?option=com_content\& view $=$ article \&id $=48 \&$ Itemid $=63$ ).

García, N., K. Cooman, 2004. Niveles de contaminación del Centro Histórico de Cuenca. En: Calidad de aire. Centro de Estudios Ambientales de la Universidad de Cuenca (http://www.ceaucuenca.ec/ index.php?option=com_content $\&$ view=article \&id=48\&Itemid=63).

Giardina, E.B., O.S. Heredia, M.A. Castro, D.N. Effron, 2012. Fitotóxicidad del cromo sobre Phaseolus vulgaris L. Descargado de ri.agro.uba.ar/files/download/revista/.../2012giardina.pdf en noviembre de 2014.

Iannacone, J., L. Alvariño, 2005. Efecto toxicológico de tres metales pesados sobre el crecimiento radicular de cuatro plantas vasculares. Agricultura Técnica (Chile), 65(2), 198-203.

Jachero L., D. Moscoso, 2009. Evaluación del riesgo de exposición a sustancias tóxicas procedentes de la contaminación atmosférica en la Ciudad de Cuenca. Centro de Estudios Ambientales de la Universidad de Cuenca. Cuenca, Ecuador (http://www.ceaucuenca.ec/index.php? option=com_content\& view $=$ article $\& i d=48 \&$ Itemid $=63$ ).

Moscoso, D., B. Pacheco, 2006. Monitoreo pasivo de la calidad de aire de la ciudad de Cuenca. En: Contaminación del aire. Centro de Estudios Ambientales. Universidad de Cuenca (http://www.ceaucuenca.ec/ index.php?option=com_content\&view=article\&id=48\&Itemid=63).

Prieto, J., C. González, A. Román, F. Prieto, 2009. Contaminación y fitotóxicidad en plantas por metales pesados provenientes de suelos y agua. Tropical and Subtropical Agroecosystems, 10(1), $29-44$.

Ribera, A., J. Canto, 2012. Mediciones de Material Particulado Sedimentable (MPS) y polvo contenido sobre los glaciares (PCG). Centro de estudios científicos para la Compañía Nevada del Proyecto Lama. Descarga de http://wp.cedha.net/wp-content/uploads/2012/11/7-Monitoreo-deMPS-y-PCG-Pascua-Lama-2012-2.pdf en octubre de 2014. 
Sierra, A., E. Simonne, D. Treadwell, 2010. Principios y prácticas de manejo de nutrientes en la producción de hortalizas. Descargado de http://edis.ifas.ufl.edu/hs356 en noviembre de 2014.

Sobrero, M., A. Ronco, 2005. Ensayo de toxicidad aguda con semillas de lechuga (Lactuca Sativa L.). Descarga de http://www2.inecc.gob.mx/publicaciones/libros/573/cap4.pdf en octubre de 2014.

Sobrero, M., 2010. Estudio de la fitotóxicidad de metales pesados y del herbicida glifosato en ambientes acuáticos. Bioensayos con plantas vasculares como organismos diagnósticos. Universidad Nacional de la Plata. Descarga de http://sedici.unlp.edu.ar/handle/10915/5246 en octubre de 2014.

TULASMA, 2002. Texto Unificado de Legislación Ambiental Secundaria del Ministerio de Ambiente: libro VI. Anexo 4. Norma de calidad de aire ambiente. Tabla 2.

Vargas, L., M. Martinez, R. Ortiz, J. López, 2007. Efectos de metales pesados sobre el crecimiento de la raíz primaria de Arabidopsis thaliana L. Facultad de Biología, Instituto de Investigaciones Químico-Biológico, UMSNH. Ciencia Nicolaita, 49, 101-112.

Varnero, M., C. Rojas, R. Orellana, 2007. Índices de fitotóxicidad en residuos orgánicos durante el compostaje. Soil Sci. Nutr., 7(1), 28-37.

Venegas, L., P. Martín, 2004. Concentración de material particulado en aire en la ciudad de Buenos Aires. Descarga de http://www.bvsde.paho.org/bvsaidis/argentinal4/venegas2.pdf en noviembre de 2014.

Wang, W., 1987. Root elongation method for toxicity testing of organic and inorganic pollutants. Environ. Toxicol. Chem., 6(5), 409-414.

\section{NOMENCLATURA}

MPS: Material particulado sedimentable, que cae por acción de la gravedad

$\mathrm{PM}_{10}$ : Conjunto de partículas que atraviesa un cabezal de tamaño selectivo para un diámetro aerodinámico de $10 \mu \mathrm{m}$ con una eficiencia de corte del $50 \%$

$\mathrm{PM}_{2.5}$ : Conjunto de partículas que atraviesa un cabezal de tamaño selectivo para un diámetro aerodinámico de $2.5 \mu \mathrm{m}$ con una eficiencia de corte del $50 \%$

$\mathrm{mg} \mathrm{cm}{ }^{-2}$ x $30 \mathrm{~d}$ : Miligramos de material por unidad de superficie, recolectado durante treinta días M (molar): Unidades de concentración que indican un mol de soluto en un litro de solución $\mathrm{mM}$ (milimolar): $10^{-3} \mathrm{M}$

$\mu \mathrm{mol}$ (micromol): $10^{-6} \mathrm{M}$

nmol (nanomol): $10^{-9} \mathrm{M}$

nmol g ${ }^{-1}$ : Nano mol por gramo de biomasa seca

$\mu \mathrm{mol} \mathrm{g} \mathrm{g}^{-1}$ : Micromol por gramo de biomasa seca

$\mathrm{mg}^{-1}$ : Miligramos de soluto por litro de solución 